\title{
The prognostic value of the lymph node ratio in patients with stage IIIC ovarian cancer treated with preoperative chemotherapy
}

\author{
Jing Hai ${ }^{1 \#}$, Li Feng ${ }^{2 \#}$, Jing Yang ${ }^{1}$, Xianyi Niu ${ }^{3}$, Hong Jiang ${ }^{4}$ \\ ${ }^{1}$ Gynecological Oncology Division 1, Department of Gynecology, Xinxiang Central Hospital, The Fourth Clinical Medical College of Xinxiang \\ Medical University, Xinxiang, China; ${ }^{2}$ Department of Oncology, No. 940 Hospital of the Joint Logistics Support Force of the Chinese People's \\ Liberation Army, Lanzhou, China; ${ }^{3}$ District 2 of the Department of Gynecology, Affiliated Hospital of Chifeng College, Chifeng City, Inner \\ Mongolia Autonomous Region, Chifeng, Inner Mongolia, China; ${ }^{4}$ Department of Obstetrics and Gynecology, Xiamen Chang Gung Hospital, \\ Xiamen, China \\ Contributions: (I) Conception and design: J Hai, H Jiang; (II) Administrative support: J Hai, H Jiang; (III) Provision of study materials or patients: All \\ authors; (IV) Collection and assembly of data: All authors; (V) Data analysis and interpretation: All authors; (VI) Manuscript writing: All authors; (VII) \\ Final approval of manuscript: All authors. \\ \#These authors contributed equally to this work. \\ Correspondence to: Hong Jiang. Department of Obstetrics and Gynecology, Xiamen Chang Gung Hospital, Xiamen, China. \\ Email: jianghong0769@sina.com.
}

Background Ovarian cancer is a major cause of morbidity and mortality in females. Due to the unremarkable symptoms associated with early ovarian cancer, many patients are already in the advanced stages at first diagnosis. Recent studies have shown that the lymph node ratio (LNR) has a certain value in predicting the prognosis of patients with ovarian cancer. However, preoperative chemotherapy may lead to changes in the LNR, and thus, the predictive value of the LNR in such patients is unclear. Therefore, this study examined the predictive value of the LNR in ovarian cancer patients undergoing preoperative chemotherapy.

Methods: A total of 208 patients with stage IIIC ovarian cancer, who were treated in the Fourth Clinical Medical College of Xinxiang Medical University, Xinxiang Central Hospital from January 2014 to January 2016, were recruited for this study. The median LNR was 0.32. Patients with LNR $<0.32$ were defined as the control group and those with $\mathrm{LNR} \geq 0.32$ were defined as the observation group. The prognosis of the two groups was compared.

Results: The LNR in the observation group was significantly higher than that of the control group $(0.63 \pm 0.21$ vs. $0.19 \pm 0.08 ; \mathrm{P}=0.000)$. Patients in the observation group showed significantly higher postoperative recurrence rates $(51.92 \%$ vs. $22.12 \% ; \mathrm{P}=0.000)$, greater incidences of postoperative metastasis (43.27\% vs. $17.31 \% ; \mathrm{P}=0.000)$, and significantly increased postoperative mortality (36.54\% vs. $11.54 \%$; $\mathrm{P}=0.000)$ compared to the control group. The survival function showed that the overall survival, recurrencefree survival, and metastasis-free survival were all significantly shorter in the observation group compared to the control group $(\mathrm{P}=0.000)$. The receiver operating characteristic curves showed that the LNR had certain diagnostic value for postoperative recurrence [area under the curve (AUC) $=0.658 ; 95 \%$ confidence interval (CI): 0.582 to $0.734 ; \mathrm{P}=0.000$ ], postoperative metastasis ( $\mathrm{AUC}=0.640 ; 95 \% \mathrm{CI}: 0.560$ to $0.720 ; \mathrm{P}=0.001$ ), and postoperative mortality ( $\mathrm{AUC}=0.653 ; 95 \% \mathrm{CI}$ : 0.569 to 0.737 ; $\mathrm{P}=0.001$ ) in patients with stage IIIC ovarian cancer treated with preoperative chemotherapy.

Conclusions: The LNR has good prognostic value in patients with locally advanced ovarian cancer treated with preoperative chemotherapy.

Keywords: Lymph node ratio (LNR); preoperative chemotherapy; ovarian cancer

Submitted Aug 17, 2021. Accepted for publication Sep 28, 2021.

doi: 10.21037/apm-21-2559

View this article at: https://dx.doi.org/10.21037/apm-21-2559 


\section{Introduction}

Ovarian cancer is the fifth leading cause of cancer-related deaths in females. As the symptoms of early stage ovarian cancer are obscure, most patients present with locally advanced ovarian cancer at the time of diagnosis, resulting in reduced survival rates (1). At present, systemic inflammation can be used to predict the prognosis of patients with ovarian cancer, but its value is limited. Previous studies have shown that lymph node status is significantly related to the survival rate of patients with ovarian cancer $(2,3)$. In fact, the Federation International of Gynecology and Obstetrics (FIGO) regards lymph node status as an important factor in the staging of ovarian cancer $(4,5)$. Lymph node metastasis has a significant impact on the prognosis of patients with ovarian cancer and such patients will require systematic lymph node resection. Lymphadenectomy has been shown to improve the prognosis and survival of patients with advanced epithelial ovarian cancer (6). The lymph node ratio (LNR) is the ratio of the number of metastatic lymph nodes to the total number of lymph nodes dissected. The LNR has certain value in predicting the prognosis of ovarian cancer, breast cancer, esophageal cancer, and other malignancies (7-15). However, preoperative chemotherapy, which is often given to patients with stage IIIC ovarian cancer, can change the number of positive lymph nodes. Therefore, this study explored the predictive and prognostic value of the LNR in patients with stage IIIC ovarian cancer undergoing preoperative chemotherapy. We present the following article in accordance with the STARD reporting checklist (available at https://dx.doi.org/10.21037/apm-21-2559).

\section{Methods}

\section{General data}

A total of 208 patients with stage IIIC epithelial ovarian cancer, who were treated in the Fourth Clinical Medical College of Xinxiang Medical University, Xinxiang Central Hospital from January 2014 to January 2016, were prospectively and continuously enrolled in this study. The median LNR was 0.32 . Patients with a $\mathrm{LNR}<0.32$ were defined as the control group $(n=104)$, and those with LNR $\geq 0.32$ were defined as the observation group $(n=104)$.

The following inclusion criteria were applied in the selection process: (I) patients diagnosed with stage IIIC epithelial ovarian cancer; (II) patients who received preoperative chemotherapy in our hospital; (III) the lymph node status could be obtained after surgical treatment;
(IV) patients were regularly followed-up for a period of 5 years after the operation to assess postoperative recurrence, metastasis, death, and other data; (V) patient were females aged 18-65 years; and (VI) more than 10 lymph nodes were cleaned during the operation.

The following exclusion criteria were applied: (I) patients who presented with other malignant tumor or major disease; (II) patients who received radiotherapy, molecular targeted therapy, or other special treatment pre-operation; (III) patients with postoperative recurrence of ovarian cancer or distant metastasis; (IV) patients with dysfunction of the liver, kidney, heart, brain, lung, or other important organ that limited their tolerance to chemotherapy or surgery; (V) patients with insufficient clinical data; and (VI) patients who were transferred to another hospital during treatment or follow-up and the follow-up data could not be obtained.

This study complies with the Declaration of Helsinki (as revised in 2013) and has been approved by the ethics committee of the Fourth Clinical Medical College of Xinxiang Medical University, Xinxiang Central Hospital (ethics approval No.: 2014-01-18). Informed consent was obtained from all patients.

\section{Treatment regimen}

All patients were given preoperative chemotherapy consisting of combined paclitaxel and carboplatin every 21 days for 3 cycles. After 3 cycles of chemotherapy, radical operation for ovarian cancer and systematic lymph node dissection was performed.

\section{Outcome measures}

Information regarding age, menstrual status, smoking history, alcohol consumption history, ovarian cancer cell differentiation, lesion location, cancer antigen (CA)125 and CA19-9 status, tumor size, ascites, postoperative recurrence, postoperative metastasis, postoperative mortality, and LNR were collated. LNR is defined as the number of positive lymph nodes divided by the total number of lymph nodes removed.

\section{Statistical analysis}

The SPSS 26.0 (IBM, Chicago, IL, USA) software was used for statistical analysis. Continuous variables conforming to normal distribution are expressed as mean \pm standard 
Table 1 The general patient characteristics

\begin{tabular}{lccccc}
\hline Group & $\mathrm{n}$ & $\begin{array}{c}\text { Age (year), } \\
\text { mean } \pm \text { SD }\end{array}$ & $\begin{array}{c}\text { Menstrual status } \\
\text { (menolipsis), } \mathrm{n}(\%)\end{array}$ & \multicolumn{2}{c}{$\begin{array}{c}\text { Smoking history, } \\
\mathrm{n}(\%)\end{array}$} \\
\hline Observation & 104 & $57.84 \pm 8.76$ & $76(73.08)$ & $12(11.54)$ & $6(5.77)$ \\
Control & 104 & $58.34 \pm 9.02$ & $79(75.96)$ & $8(7.69)$ & $9(8.65)$ \\
$t / \chi^{2}$ value & & 0.406 & 0.228 & 0.885 & 0.647 \\
$P$ value & 0.686 & 0.663 & 0.347 & 0.421 \\
\hline
\end{tabular}

SD, standard deviation.

Table 2 Basic clinical characteristics of the patients

\begin{tabular}{lccccc}
\hline Group & $\mathrm{n}$ & Lesion location (left), $\mathrm{n}(\%)$ & Ascites, $\mathrm{n}(\%)$ & $\mathrm{CA125}(\mathrm{U} / \mathrm{mL}), \mathrm{mean} \pm \mathrm{SD}$ & $\mathrm{CA} 19-9(\mathrm{U} / \mathrm{mL}), \mathrm{mean} \pm \mathrm{SD}$ \\
\hline Observation & 104 & $65(62.50)$ & $48(46.15)$ & $93.27 \pm 12.74$ & $135.39 \pm 24.59$ \\
Control & 104 & $60(57.69)$ & $43(41.35)$ & $86.48 \pm 10.72$ & $118.85 \pm 31.48$ \\
$t / \chi^{2}$ value & 0.501 & 0.488 & 4.159 & 4.223 \\
$P$ value & 0.479 & 0.485 & 0.000 & 0.000 \\
\hline
\end{tabular}

CA125, carbohydrate antigen 125; CA19-9, carbohydrate antigen 19-9.

deviation and the data from the two groups were analyzed by independent sample $t$-test. The count data of the two groups are expressed as $\mathrm{n}(\%)$ and analyzed by the chi-square test. Survival function and chi-square tests were used to analyze the differences of postoperative recurrence, postoperative metastasis, and postoperative mortality between the two groups. Receiver operating characteristic (ROC) curves were used to analyze the diagnostic value of the LNR in postoperative recurrence, postoperative metastasis, and postoperative mortality of patients with stage IIIC ovarian cancer treated with preoperative chemotherapy.

\section{Results}

\section{General patient characteristics}

Patients with a LNR $<0.32$ were defined as the control group ( $n=104)$, and those with LNR $\geq 0.32$ were defined as the observation group $(n=104)$. There were no statistically significant differences in the general data between the two groups, including age, menstrual status, and smoking and drinking history $(\mathrm{P}>0.05 ;$ Table 1$)$.

\section{Patient clinical characteristics}

There were no significant differences in the lesion location nor the incidence of ascites between the observation group and the control group $(\mathrm{P}>0.05)$. However, the levels of the cancer antigens CA125 and CA19-9 in patients in the observation group were significantly increased compared to the levels observed in patients from the control group $(\mathrm{P}<0.05$; Table 2).

\section{Patbological characteristics of the patients}

There were no significant differences in the grade of tumor cell differentiation between the two groups $(\mathrm{P}>0.05)$. However, the tumor size in the observation group was significantly greater than that in the control group $(5.23 \pm 1.95$ vs. $4.38 \pm 1.62 \mathrm{~cm} ; \mathrm{P}=0.001)$, and the $\mathrm{LNR}$ was also significantly elevated in the observation group compared to the control group $(0.63 \pm 0.21$ vs. $0.19 \pm 0.08$; $\mathrm{P}=0.000$; Table 3).

\section{Postoperative follow-up of patients}

The postoperative recurrence rate was significantly higher in the observation group compared to the control group (51.92\% vs. $22.12 \% ; \mathrm{P}=0.000)$. Similarly, both the postoperative metastasis rate $(43.27 \%$ vs. $17.31 \% ; \mathrm{P}=0.000)$ and the postoperative mortality rate were significantly higher in the observation group compared to the control group (36.54\% vs. $11.54 \% ; \mathrm{P}=0.000)$. The survival function showed that the overall survival, recurrence-free survival, 
Table 3 The pathological characteristics of the patients

\begin{tabular}{lcccc}
\hline Group & $\mathrm{n}$ & Grade of differentiation (middle or low differentiation), $\mathrm{n}(\%)$ & Tumor size $(\mathrm{cm})$, mean \pm SD & LNR, mean \pm SD \\
\hline Observation & 104 & $76(73.08)$ & $5.23 \pm 1.95$ & $0.63 \pm 0.21$ \\
Control & 104 & $67(64.42)$ & $4.38 \pm 1.62$ & $0.19 \pm 0.08$ \\
$t / \chi^{2}$ value & & 1.813 & 3.419 & 19.946 \\
$P$ value & & 0.178 & 0.001 & 0.000 \\
\hline
\end{tabular}

LNR, lymph node ratio; SD, standard deviation.

Table 4 Postoperative follow-up of patients

\begin{tabular}{|c|c|c|c|c|}
\hline Group & $\mathrm{n}$ & Recurrence, n (\%) & Metastasis, n (\%) & Mortality, n (\%) \\
\hline Control & 104 & $23(22.12)$ & $18(17.31)$ & $12(11.54)$ \\
\hline$\chi^{2}$ value & & 19.816 & 16.599 & 17.798 \\
\hline$P$ value & & 0.000 & 0.000 & 0.000 \\
\hline
\end{tabular}

and metastasis-free survival in the observation group were all significantly shortened compared to the control group $(\mathrm{P}=0.000 ;$ Table 4 and Figures 1-3).

\section{Diagnostic value of the LNR on postoperative recurrence, metastasis, and death in patients with stage IIIC ovarian cancer treated with preoperative chemotherapy}

The LNR has certain diagnostic value on the postoperative recurrence, metastasis, and death in patients with stage IIIC ovarian cancer treated with preoperative chemotherapy. The areas under the ROC curves for postoperative recurrence, metastasis, and mortality were 0.658 [95\% confidence interval (CI): 0.582 to $0.734 ; \mathrm{P}=0.000), 0.640$ (95\% CI: 0.560 to 0.720 ); $\mathrm{P}=0.001$ ] , and 0.653 (95\% CI: 0.569 to 0.737 ; $\mathrm{P}=0.001$ ), respectively (Table 5 and Figures 4-6).

\section{Diagnostic value of number of lymph node metastases on postoperative recurrence, metastasis, and death in patients with stage IIIC ovarian cancer treated with preoperative chemotherapy}

The number of lymph node metastases has certain diagnostic value on the postoperative recurrence, metastasis, and death in patients with stage IIIC ovarian cancer treated with preoperative chemotherapy. The areas under the ROC curves for postoperative recurrence, metastasis, and mortality were 0.653 (95\% CI: 0.576-0.729; P=0.000),
0.625 (95\% CI: 0.544-0.707; P=0.004), and 0.626 (95\% CI: $0.540-0.712 ; \mathrm{P}=0.007)$, respectively (Figures $7-9$ ).

\section{Discussion}

The pathogenic factors of ovarian tumors are unclear, but the environmental and endocrine effects are the most important among the pathogenic factors of ovarian tumors. Studies to date have confirmed that the LNR has good predictive value for the prognosis of patients with malignant tumors, including ovarian cancers (12-20). Preoperative chemotherapy has a certain impact on the lymph node status of patients with malignant tumors. Some patients with preoperative chemotherapy can achieve a complete pathological response, which can impact the prognostic value of the LNR (21-24). Unfortunately, there is currently a paucity of data examining the predictive value of the LNR in patients with stage IIIC ovarian cancer treated with preoperative chemotherapy. This current report demonstrated that in ovarian cancer patients with preoperative chemotherapy, a high LNR was associated with significantly elevated postoperative recurrence rates, postoperative metastasis rates, and postoperative mortality. Furthermore, the overall survival, recurrence-free survival, and metastasis-free survival were significantly shortened in patients with a high LNR. Therefore, the LNR was significantly associated with postoperative recurrence, metastasis, and mortality in patients with stage IIIC ovarian cancer treated with preoperative chemotherapy. The areas 


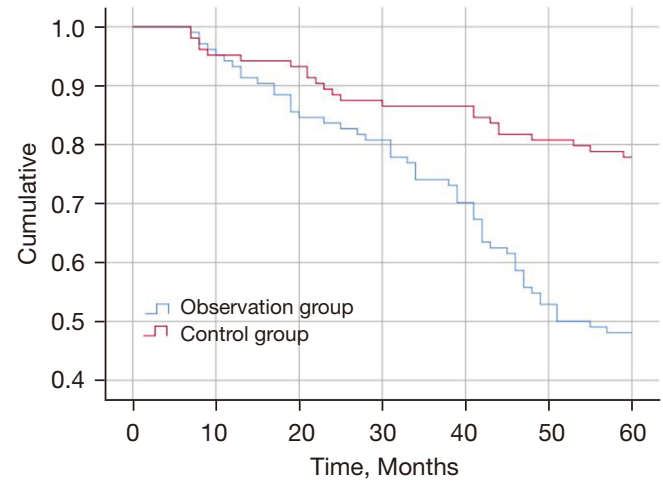

Figure 1 A comparison of the 5-year recurrence-free rate between the observation group and the control group (survival function: Wilcoxon test value $=16.490 ; \mathrm{P}=0.000)$.

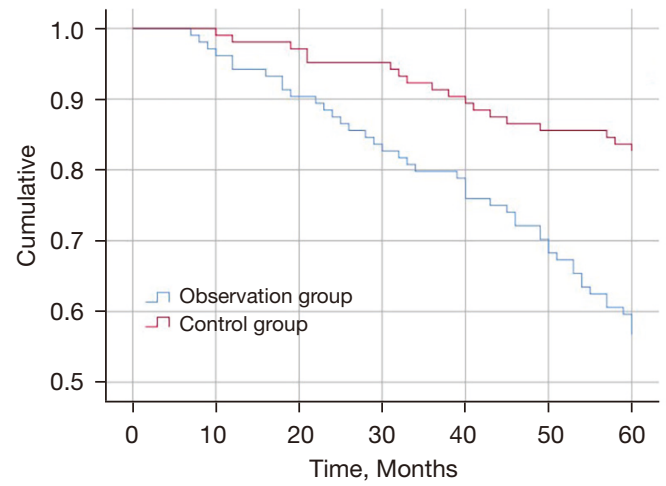

Figure 2 A comparison of the 5-year metastasis-free rate between the observation group and the control group (survival function: Wilcoxon test value $=15.512, \mathrm{P}=0.000$ ).

under the curve were 0.658 (95\% CI: 0.582 to 0.734 ; $\mathrm{P}=0.000), 0.640$ (95\% CI: 0.560 to $0.720 ; \mathrm{P}=0.001)$, and 0.653 (95\% CI: 0.569 to $0.737 ; \mathrm{P}=0.001$ ), respectively.

Lymphatic metastasis is a significant metastatic pathway of ovarian cancer. The tumor cells can go up to the paraaortic lymph nodes along the ovarian vessels, through the ovarian lymphatic vessels. It can also reach the internal and external iliac lymph nodes along the lymphatic vessels of the ovarian portal, and pass through the lymph nodes near the common iliac artery to the lymph nodes near the abdominal aorta. Chan et al. analyzed the data of 6,686 patients with stage I ovarian cancer. The 5 -year survival rate of patients who underwent systematic lymphadenectomy was significantly improved $(93.3 \% \mathrm{vs}$. $85.9 \% ; \mathrm{P}<0.001)(25)$. Patients with simple lymph node recurrence after ovarian cancer surgery can be operated

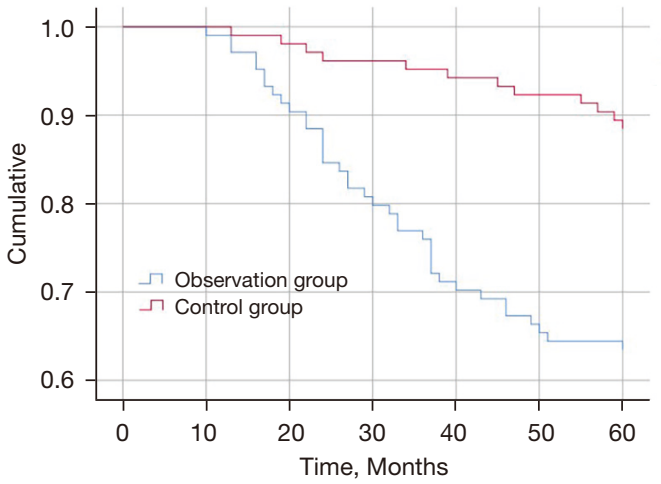

Figure 3 A comparison of the 5-year survival rate between the observation group and the control group (survival function: Wilcoxon test value $=19.273, \mathrm{P}=0.000$ ) .

again. At the same time, postoperative radiotherapy and chemotherapy may be helpful to improve the prognosis of the patients. Ovarian cancer patients with lymph node metastasis are classified as stage IIIC by the FIGO staging system $(4,5)$. The LNR is the ratio of the number of positive lymph nodes to the total number of lymph nodes cleaned during the operation. Therefore, patients with higher LNR have more lymph nodes that have been invaded with ovarian cancer, and thus, are more likely to relapse, metastasize, or die post-operatively $(26,27)$. Studies have shown that LNR $\geq 0.42$ is an independent risk factor for death in patients with ovarian cancer and this value has particular significant in predicting the prognosis of patients (12). A Turkish study involving 229 patients with stage IIIC ovarian cancer divided the patients as follows: the LNR $<10 \%$ group, the $10 \% \leq \mathrm{LNR}$ $<50 \%$ group, and the LNR $\geq 50 \%$ group. The results showed that the 5 -year overall survival rates of the three groups were $65.1 \%, 42.5 \%$, and $25.6 \%$, respectively $(\mathrm{P}<0.001)$. Thus, increased LNR was an independent risk factor for mortality in patients with ovarian cancer (13). Another study based on the Surveillance, Epidemiology, and End Results (SEER) database showed that LNR had strong prognostic value in patients with ovarian cancer after stratification according to the number of lymph nodes, tumor histology, and tumor grade (14). Another study showed that LNR $>0.25$ was an independent risk factor for reduced overall survival (15). In agreement with these previous studies, this current investigation examining stage IIIC ovarian cancer patients undergoing preoperative chemotherapy, confirmed that the LNR has good prognostic value in such patients. Of course, we can 
Table 5 The diagnostic value of the LNR on postoperative recurrence, metastasis, and mortality in patients with stage IIIC ovarian cancer treated with preoperative chemotherapy

\begin{tabular}{lcccc}
\hline Indicator & Area under the curve & Standard error & $\mathrm{P}$ value & $95 \% \mathrm{Cl}$ \\
\hline Recurrence rate & 0.658 & 0.039 & 0.000 & $0.582-0.734$ \\
Metastasis rate & 0.640 & 0.041 & 0.001 & $0.560-0.720$ \\
Mortality & 0.653 & 0.043 & 0.001 & $0.569-0.737$ \\
\hline
\end{tabular}

LNR, lymph node ratio; $\mathrm{Cl}$, confidence interval.

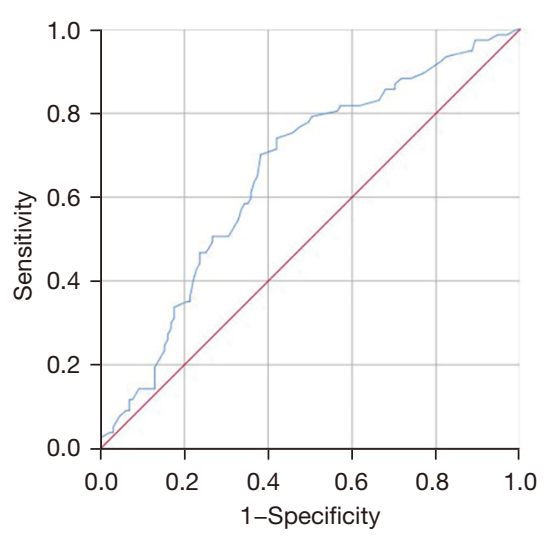

Figure 4 The diagnostic value of the lymph node ratio (LNR) in postoperative recurrence of stage IIIC ovarian cancer treated with preoperative chemotherapy.

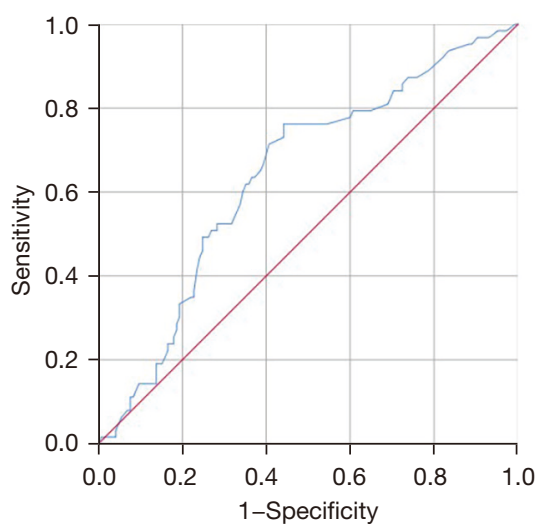

Figure 5 The diagnostic value of the lymph node ratio (LNR) in postoperative metastasis of stage IIIC ovarian cancer treated with preoperative chemotherapy.

also use gene differences to predict the prognosis of patients with ovarian cancer, such as inflammation related genes. But these are in urgent need of further research.

This study was limited by the small sample size which

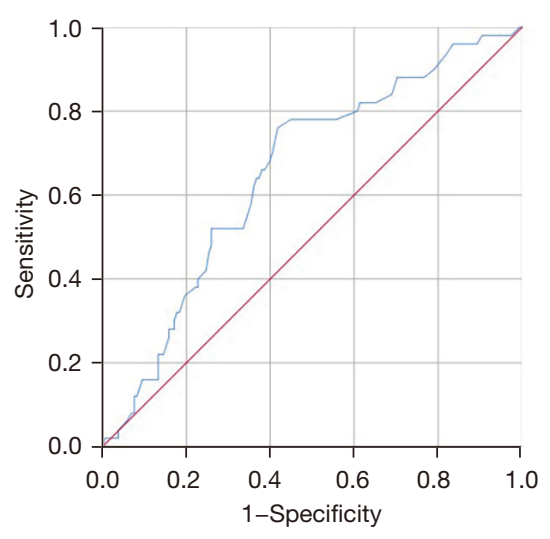

Figure 6 The diagnostic value of the lymph node ratio (LNR) in postoperative death of patients with stage IIIC ovarian cancer treated with preoperative chemotherapy.

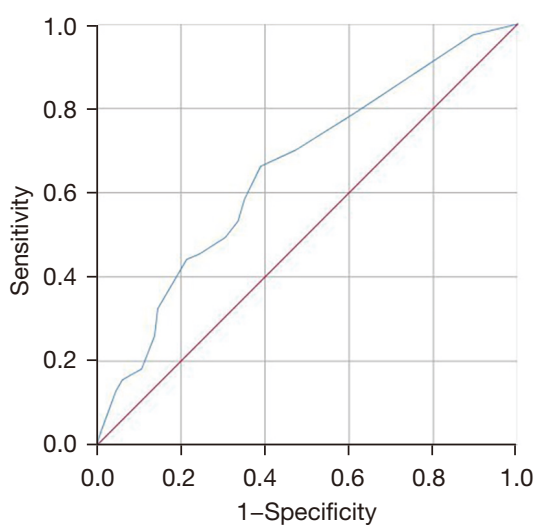

Figure 7 The diagnostic value of number of lymph node metastases in postoperative recurrence of stage IIIC ovarian cancer treated with preoperative chemotherapy.

was insufficient to conduct stratified analysis on ovarian cancer patients of different ages and different grades of differentiation. Future work should include larger sample sizes to further understand the prognostic role of the LNR. 


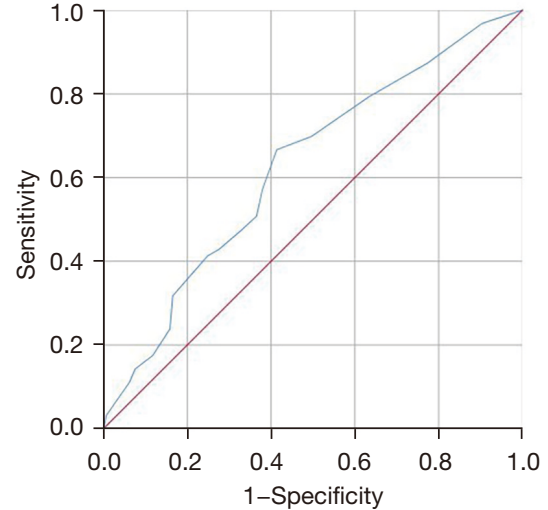

Figure 8 The diagnostic value of number of lymph node metastases in postoperative recurrence of stage IIIC ovarian cancer treated with preoperative chemotherapy.

\section{Acknowledgments}

Funding: None.

\section{Footnote}

Reporting Checklist: The authors have completed the STARD reporting checklist. Available at https://dx.doi. org/10.21037/apm-21-2559

Data Sharing Statement: Available at https://dx.doi. org/10.21037/apm-21-2559

Conflicts of Interest: All authors have completed the ICMJE uniform disclosure form (available at https://dx.doi. org/10.21037/apm-21-2559). The authors have no conflicts of interest to declare.

Ethical Statement: The authors are accountable for all aspects of the work in ensuring that questions related to the accuracy or integrity of any part of the work are appropriately investigated and resolved. This study was conducted in accordance with the Declaration of Helsinki (as revised in 2013) and has been approved by the ethics committee of the Fourth Clinical Medical College of Xinxiang Medical University, Xinxiang Central Hospital (ethics approval No.: 2014-01-18). Informed consent was obtained from all patients.

Open Access Statement: This is an Open Access article distributed in accordance with the Creative Commons

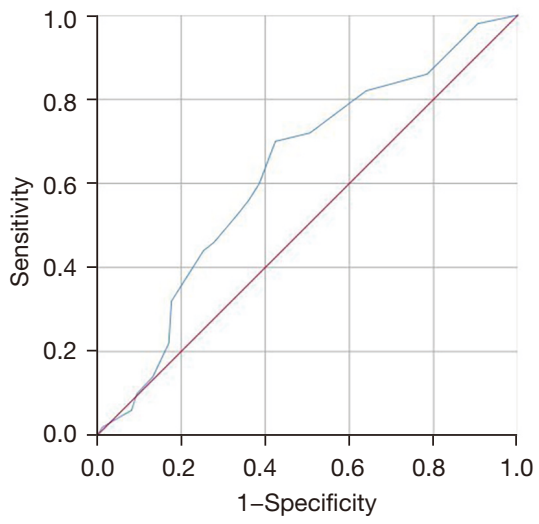

Figure 9 The diagnostic value of number of lymph node metastases in postoperative recurrence of stage IIIC ovarian cancer treated with preoperative chemotherapy.

Attribution-NonCommercial-NoDerivs 4.0 International License (CC BY-NC-ND 4.0), which permits the noncommercial replication and distribution of the article with the strict proviso that no changes or edits are made and the original work is properly cited (including links to both the formal publication through the relevant DOI and the license). See: https://creativecommons.org/licenses/by-nc-nd/4.0/.

\section{References}

1. Siegel RL, Miller KD, Fuchs HE, et al. Cancer Statistics, 2021. CA Cancer J Clin 2021;71:7-33.

2. du Bois A, Reuss A, Harter P, et al. Potential role of lymphadenectomy in advanced ovarian cancer: a combined exploratory analysis of three prospectively randomized phase III multicenter trials. J Clin Oncol 2010;28:1733-9.

3. Yuan Y, Wang R, Guo F, et al. A clinical model to predict the risk of liver metastases in newly diagnosed ovarian cancer: a population-based study. Transl Cancer Res 2020;9:7044-53.

4. Prat J. Ovarian, fallopian tube and peritoneal cancer staging: Rationale and explanation of new FIGO staging 2013. Best Pract Res Clin Obstet Gynaecol 2015;29:858-69.

5. Zeppernick F, Meinhold-Heerlein I. The new FIGO staging system for ovarian, fallopian tube, and primary peritoneal cancer. Arch Gynecol Obstet 2014;290:839-42.

6. Gao J, Yang X, Zhang Y. Systematic lymphadenectomy in the treatment of epithelial ovarian cancer: a metaanalysis of multiple epidemiology studies. Jpn J Clin Oncol 
2015;45:49-60.

7. Melis M, Masi A, Pinna A, et al. Does lymph node ratio affect prognosis in gastroesophageal cancer? Am J Surg 2015;210:443-50.

8. Philip A, Jose M, Jose WM, et al. Pretreatment neutrophilto-lymphocyte ratio predicts lymph node metastasis in triple-negative breast cancer. Indian J Cancer 2021. [Epub ahead of print]. doi: 10.4103/ijc.IJC_914_19.

9. Vinh-Hung V, Everaert H, Gorobets O, et al. Breast cancer preoperative 18FDG-PET, overall survival prognostic separation compared with the lymph node ratio. Breast Cancer 2021;28:956-68.

10. Zhong R, Jiang X, Peng Y, et al. A nomogram prediction of overall survival based on lymph node ratio, AJCC 8th staging system, and other factors for primary pancreatic cancer. PLoS One 2021;16:e0249911.

11. Peng JS, Morris-Stiff G, Ali NS, et al. Neoadjuvant chemoradiation is associated with decreased lymph node ratio in borderline resectable pancreatic cancer: A propensity score matched analysis. Hepatobiliary Pancreat Dis Int 2021;20:74-9.

12. Tong X, Li H, Chen H, et al. Prognostic Significance of Lymph Node Ratio in Ovarian Cancer. Open Med (Wars) 2019;14:279-86.

13. Ayhan A, Ozkan NT, Sarı ME, et al. Impact of lymph node ratio on survival in stage III ovarian high-grade serous cancer: a Turkish Gynecologic Oncology Group study. J Gynecol Oncol 2018;29:e12.

14. Zhou J, He ZY, Li FY, et al. Prognostic value of lymph node ratio in stage IIIC epithelial ovarian cancer with node-positive in a SEER population-based study. Oncotarget 2016;7:7952-9.

15. Ataseven B, Grimm C, Harter P, et al. Prognostic value of lymph node ratio in patients with advanced epithelial ovarian cancer. Gynecol Oncol 2014;135:435-40.

16. De la Cruz-Ku GA, Chambergo-Michilot D, Valcarcel B, et al. Lymph node ratio as best prognostic factor in triplenegative breast cancer patients with residual disease after neoadjuvant chemotherapy. Breast J 2020;26:1659-66.

17. Kaul P, Panuganti A, Thaduri A, et al. Lymph node ratio in oral cavity cancer and its impact on mortality and disease recurrence. Oral Oncol 2020;111:104880.

18. Yukawa N, Aoyama T, Tamagawa H, et al. The Lymph Node Ratio Is an Independent Prognostic Factor in Esophageal Cancer Patients Who Receive Curative Surgery. In Vivo 2020;34:2087-93.

19. Chen PJ, Yap WK, Chang YC, et al. Prognostic value of lymph node to primary tumor standardized uptake value ratio in unresectable esophageal cancer. BMC Cancer 2020;20:545.

20. Kano K, Yamada T, Oshima T. ASO Author Reflections: Association Between the Lymph Node Ratio and Survival in Patients with Pathological Stage II/III Gastric Cancer. Ann Surg Oncol 2020;27:4248-9.

21. Flores P, Cadario M, Strambach J, et al. Outcomes of event-free survival in patients with Wilms tumor undergoing preoperative chemotherapy. Analysis of lymph-node yield in a single-center cohort. J Pediatr Urol 2021;17:227.e1-e5.

22. Swords DS, Francis SR, Lloyd S, et al. Lymph Node Ratio in Pancreatic Adenocarcinoma After Preoperative Chemotherapy vs. Preoperative Chemoradiation and Its Utility in Decisions About Postoperative Chemotherapy. J Gastrointest Surg 2019;23:1401-13.

23. Takada K, Kashiwagi S, Goto W, et al. Possibility of avoiding axillary lymph node dissection by immune microenvironment monitoring in preoperative chemotherapy for breast cancer. J Transl Med 2018;16:318.

24. Yamashita K, Hosoda K, Moriya H, et al. Prognostic Advantage of Docetaxel/Cisplatin/ 5-Fluorouracil Neoadjuvant Chemotherapy in Clinical Stage II/III Esophageal Squamous Cell Carcinoma due to Excellent Control of Preoperative Disease and Postoperative Lymph Node Recurrence. Oncology 2017;92:221-8.

25. Chan JK, Munro EG, Cheung MK, et al. Association of lymphadenectomy and survival in stage I ovarian cancer patients. Obstet Gynecol 2007;109:12-9.

26. Tanaka K, Shimada Y, Nishino K, et al. Clinical Significance of Mesenteric Lymph Node Involvement in the Pattern of Liver Metastasis in Patients with Ovarian Cancer. Ann Surg Oncol 2021. [Epub ahead of print]. doi: 10.1245/s10434-021-09899-8.

27. Lee IO, Lee JY, Kim HJ, et al. Prognostic significance of supradiaphragmatic lymph node metastasis detected by 18F-FDG PET/CT in advanced epithelial ovarian cancer. BMC Cancer 2018;18:1165.

(English Language Editor: J. Teoh)

Cite this article as: Hai J, Feng L, Yang J, Niu X, Jiang H. The prognostic value of the lymph node ratio in patients with stage IIIC ovarian cancer treated with preoperative chemotherapy. Ann Palliat Med 2021;10(11):11504-11511. doi: 10.21037/apm-212559 